\title{
Mosquito host preferences affect their response to synthetic and natural odour blends
}

\author{
Annette O Busula ${ }^{1,2}$, Willem Takken², Dorothy E Loy ${ }^{3}$, Beatrice H Hahn³, Wolfgang R Mukabana ${ }^{1,4}$ \\ and Niels O Verhulst ${ }^{2 *}$
}

\begin{abstract}
Background: The anthropophilic malaria mosquito Anopheles gambiae sensu stricto (hereafter termed Anopheles gambiae) primarily takes blood meals from humans, whereas its close sibling Anopheles arabiensis is more opportunistic. Previous studies have identified several compounds that play a critical role in the odour-mediated behaviour of An. gambiae. This study determined the effect of natural and synthetic odour blends on mosquitoes with different host preferences to better understand the host-seeking behaviour of mosquitoes and the potential of synthetic odour blends for standardized monitoring.
\end{abstract}

Methods: Odour blends were initially tested for their attractiveness to An. gambiae and An. arabiensis in a semi-field system with MM-X traps baited with natural and synthetic odours. Natural host odours were collected from humans, cows and chickens. The synthetic odour blends consisted of three or five previously identified compounds released with carbon dioxide. These studies were continued under natural conditions where odour blends were tested outdoors to determine their effect on species with different host preferences.

Results: In the semi-field experiments, human odour attracted significantly higher numbers of both mosquito species. However, An. arabiensis was also attracted to cow and chicken odours, which confirms its opportunistic behaviour. A five-component synthetic blend was highly attractive to both mosquito species. In the field, the synthetic odour blend caught significantly more An. funestus than traps baited with human odour, while no difference was found for An. arabiensis. Catches of An. arabiensis and Culex spp. contained large numbers of blood-fed mosquitoes, mostly from cows, which indicates that these mosquitoes had fed outdoors.

Conclusions: Different odour baits elicit varying responses among mosquito species. Synthetic odour blends are highly effective for trapping mosquitoes; however, not all mosquitoes respond equally to the same odour blend. Combining fermenting molasses with synthetic blends in a trap represents the most effective tool to catch blood-fed mosquitoes outside houses, which is essential for understanding outdoor malaria transmission.

Keywords: Anopheles gambiae, Anopheles funestus, Anopheles arabiensis, Carbon dioxide, Host seeking, Attraction, Trapping, Monitoring, Anthropophilic

\section{Background}

The host preference of a mosquito species is an important determinant of its vectorial capacity and mosquito species that are highly anthropophilic are often vectors of important human diseases [1]. The anthropophilic malaria mosquitoes Anopheles gambiae s.s. and Anopheles funestus s.s., for example, primarily take blood meals

\footnotetext{
*Correspondence: niels.verhulst@wur.nl

2Laboratory of Entomology, Wageningen University, PO Box 8031, $6700 \mathrm{EH}$,

Wageningen, The Netherlands

Full list of author information is available at the end of the article
}

on humans [2] and are two of the most important malaria vectors in Africa [3]. Anopheles arabiensis, a close relative of $A n$. gambiae, is more opportunistic, feeding on both humans and animals, and is considered a less important malaria vector $[1,4]$. This difference in host preference is most evident in odour-guided behaviour, where $A n$. arabiensis responds more strongly to carbon dioxide $\left(\mathrm{CO}_{2}\right)$ as a general cue to find a host and $A n$. gambiae mainly relies on specific human odours [1].

$\mathrm{CO}_{2}$ is a major constituent of exhaled air and has been identified as an attractant for many mosquito species 
including the main vectors of malaria in Africa [5]. Gillies [6] suggested that this compound acts as an activator, initiating flight responses as well as being an attractant. There is strong evidence that $\mathrm{CO}_{2}$ acts synergistically with other chemical compounds to attract host-seeking mosquitoes [7-11], which can be used in odour-baited traps in which $\mathrm{CO}_{2}$ and synthetic blends that mimic human odour are combined $[10,12]$. These traps can then be used for monitoring, but can also intercept and reduce the number of malaria mosquitoes entering or leaving houses [9]. A standard synthetic blend (SB) consisting of $\mathrm{CO}_{2}$, ammonia, (S)-lactic acid, tetradecanoic acid was tested along with an extended blend to which 3-methyl-1-butanol and butan-1-amine (MB5 blend) was added, and found to be efficient for trapping the malaria mosquito An. gambiae in a semifield setting as well as in two traditional villages in western Kenya $[10,13,14]$. These attractive blends have been developed for anthropophilic An. gambiae mosquitoes $[10,11,14-17]$. However, less is known about their effect on the host-seeking behaviour of other mosquito species with different host preferences.

In this study, natural host odours and synthetic odour blends were dispensed from mosquito traps to determine the efficacy of synthetic blends for monitoring mosquito species with different host preferences. Anopheles gambiae and $A n$. arabiensis mosquitoes, which are reported to be anthropophilic and opportunistic respectively $[1,4]$, were simultaneously released in a semi-field system in western Kenya to determine their host-seeking behaviour, either in the presence of $\mathrm{CO}_{2}$ alone, or combined with natural odours or the synthetic blends (SB and MB5). In a field trial the efficacy of traps baited with natural odours or a synthetic blend was compared to determine the efficacy of the blend for different species of wild mosquitoes.

\section{Methods \\ Mosquitoes}

The semi-field experiments utilized laboratory colonies of the Mbita strain of An. gambiae sensu stricto and $A n$. arabiensis. Aquatic stages of the mosquitoes were separately reared under ambient atmospheric conditions in screen-walled greenhouses at the Thomas Odhiambo Campus Odhiambo (TOC) of the International Centre of Insect Physiology and Ecology (ICIPE), Mbita, Kenya. Adult mosquitoes were placed in a holding room under ambient conditions with a scotophase of 12:12 h. Female adult mosquitoes were fed three times a week on a human arm [18]. Eggs were laid on moist filter paper and dispensed into plastic trays containing filtered water from Lake Victoria. Newly hatched larvae were transferred into plastic basins and fed on Tetramin baby fish food (Melle, Germany) three times a day. Collection of pupae until adult emergence is described in Mukabana et al. [10]. Female mosquitoes used for semi-field experiments were placed in mosquito netting covered plastic cups [10]. They had no prior access to a blood meal but were fed only on water, provided on wet cotton towels placed on top of mosquito-holding cups during starvation [10]. All semifield experiments were carried out at night (20:00-06:30 h) inside a $7 \times 11 \mathrm{~m}$ screenhouse [16]. Two-hundred females of An. gambiae and 200 An. arabiensis aged three to eight days old were painted with either pink or yellow fluorescent dyes (FTX Series, Astral Pink, Swada, London) ten hours before the experiments, as described before [19]. Mosquitoes were starved for eight hours and simultaneously released at the centre of a screen-walled greenhouse.

\section{Study sites}

Semi-field experiments were conducted between February and April 2013 in a $7 \times 11 \mathrm{~m}$ screenhouse constructed on the grounds of the TOC of ICIPE, Kenya $\left(00^{0} 25^{1} \mathrm{~S}, 34^{\circ}\right.$ $13^{1} \mathrm{E}$ ). Field studies were conducted between May and June 2013 at Kigoche village, situated near Ahero town, in the Kano plains of Kisumu County, Kenya $\left(00^{\circ} 34^{\prime}\right.$ S, $34^{\circ} 65^{\prime}$ E) $[10,20]$. The area receives between 1,000 and $1,800 \mathrm{~mm}$ of rainfall annually with annual temperature and relative humidity (RH) ranges of $17-32^{\circ} \mathrm{C}$ and $44-80 \%$, respectively. The long rainy season occurs between March and August while short rains are common in October to November. The main economic activity is rice farming which creates numerous mosquito larval habitats resulting in high malaria transmission. Indigenous goats, cattle, poultry, and sheep are also kept in Kigoche [18]. During the night, domestic animals are tethered outdoors adjacent to houses occupied by humans. Many houses in the area are mud-walled with roofs made of corrugated iron sheets or thatch, or without ceiling. Eaves of most houses are open due to the high daytime temperatures [21]. Previous studies reported that the annual Entomological Inoculation Rate (EIR) was 416 and An. arabiensis and An. funestus s.l. the main malaria vectors $[10,22,23]$.

\section{Collection of natural host odours and preparation of $\mathrm{CO}_{2}$}

Human foot odour previously shown to be moderately attractive to mosquitoes [24] was collected from nylon socks worn by a Kenyan male (age 31) (Additional file 1: Figure S1). The socks were worn for 24 hours before they were used in the experiment [25]. The volunteer did not smoke, use alcohol, spicy food, perfumes and the last shower was without soap $[24,26]$. Animal odours were collected from the same individual throughout the experiments by wrapping a clean nylon sock above the knee of a cow or around the leg of a chicken for 24 hours (Additional file 1: Figure S1). For the cow odour sample, a piece of cloth was wrapped over the sock to prevent dirt or faeces from contaminating the odour sample. 
Clean latex gloves were worn to avoid contamination by other odours. Henceforth, human, cow and chicken skin emanations collected on nylon socks will be termed "human odour", "cow odour" and "chicken odour", respectively.

Sugar and molasses were used to produce $\mathrm{CO}_{2}$ in semifield and field experiments respectively. Sugar-produced $\mathrm{CO}_{2}$ was prepared by mixing $250 \mathrm{~g}$ sugar (Mumias Sugar Co Ltd, Kenya), 17.5 g yeast (Angel ${ }^{\circ}$ Company, China) and $2 \mathrm{~L}$ water in 5-L containers which would result in an average $\mathrm{CO}_{2}$ production of $242.3 \pm 74.1 \mathrm{ml} / \mathrm{min}$ [27]. Molassesproduced $\mathrm{CO}_{2}$ was obtained by mixing $2 \mathrm{~L}$ water, $250 \mathrm{~g}$ molasses (Mumias Sugar Co Ltd, Kenya) and $17.5 \mathrm{~g}$ dry instant yeast in $5 \mathrm{~L}$ containers [18]. Tap water was used during semi-field experiments while all field bioassays were conducted using clean water from Kigoche village. Released $\mathrm{CO}_{2}$ was delivered through a $60-\mathrm{cm}$ long silicon tubing $(0.5 \mathrm{~cm}$ diameter) into individual MM-X traps (American Biophysics, North Kingstown, RI, USA) [18]. The MB5 and the SBs used in the current study were separately prepared following protocols described before $[10,13]$. Socks containing cow, chicken and human odour, and synthetic blends were separately hooked on a wire ring and hung inside the plume tube of a MM-X trap and always supplied with $\mathrm{CO}_{2}$ from either molasses or sugar. Control traps were baited with $\mathrm{CO}_{2}$ alone unless indicated specifically. The lower end of the plume tube was suspended $15 \mathrm{~cm}$ above ground level [28]. Socks and synthetic blends were placed in glass jars, and stored in a freezer until and between experiments and replaced after four experiments.

\section{General experimental procedures}

All MM-X traps were operated using a $12-\mathrm{V}$ battery. Vaseline pure petroleum jelly was applied on suspension wire bars, electrical cables and $\mathrm{CO}_{2}$ tubing to prevent ants from preying on mosquitoes caught in the MM-X traps. To terminate experiments, a plug was inserted into the outer tube of the MM-X trap, the $\mathrm{CO}_{2}$ supply was cut off, and the power disconnected [18]. Traps containing mosquitoes were placed in a refrigerator at $-4^{\circ} \mathrm{C}$ for $10 \mathrm{~min}$. Immobilized mosquitoes were collected, counted, and recorded. Traps were cleaned between experiments using $70 \%$ ethanol (to remove residual odours). A manual, handheld aspirator was used to collect untrapped, free-flying mosquitoes from the screenhouse. The sand-filled floor of the greenhouse was moistened daily to enhance survival of mosquitoes. Latex gloves were worn during experiments to avoid contamination with human volatiles or other odorant compounds.

Attractiveness of natural host odours to laboratory-reared Anopheles gambiae and Anopheles arabiensis

MM-X traps were placed in all four corners of the screenhouse, and rotated with identical treatments placed at opposite corners of the house. A total of 8 replicates (for a total of 4 nights) were carried out. The treatment combination included: (i) $\mathrm{CO}_{2}$ vs no stimulus; (ii) cow odour + $\mathrm{CO}_{2}$ vs clean sock $+\mathrm{CO}_{2}$; (iii) chicken odour $+\mathrm{CO}_{2}$ vs clean sock $+\mathrm{CO}_{2}$; and, (iv) human odour $+\mathrm{CO}_{2}$ vs clean sock $+\mathrm{CO}_{2}$.

\section{Attractiveness of natural host odours to Anopheles gambiae and Anopheles arabiensis by competition}

Randomized $4 \times 4$ Latin square experimental design was adopted. MM-X traps were placed in all four corners of the screenhouse and treatments rotated for 4 consecutive nights. A total of 16 replicates were carried out. The treatment combination included: (i) only $\mathrm{CO}_{2}$ and clean sock (control); (ii) cow odour $+\mathrm{CO}_{2}$; (iii) chicken odour $+\mathrm{CO}_{2}$; and, (iv) human odour $+\mathrm{CO}_{2}$.

\section{Attractiveness of synthetic odour blends to Anopheles gambiae and Anopheles arabiensis}

Randomized $4 \times 4$ Latin square experimental design was adopted. MM-X traps were placed in all four corners of the screenhouse and treatments rotated for four consecutive nights. A total of 12 replicates were carried out. The treatment combination included: (i) only clean nylon strips without $\mathrm{CO}_{2}$ (control); (ii) clean nylon strips $+\mathrm{CO}_{2}$; (iii) Simple Blend (SB: NH3 + Lactic acid + C14, [15]) $+\mathrm{CO}_{2}$; and, (iv) Mbita blend (MB5: NH3 + Lactic acid + C14 + 3methyl-1-butanol + Butan-1-amine [13,14]) $+\mathrm{CO}_{2}$.

\section{Response of wild mosquitoes with different host preferences to natural and synthetic odour blends}

Five village houses were selected and experiments were carried out from 18.30 to $06.30 \mathrm{~h}$ each night. Randomized $5 \times 5$ Latin square experimental design was adopted. One MM-X trap was placed at each house and treatments rotated for five consecutive nights. A total of 25 replicates were carried out. The treatment combination included a MM-X trap with $\mathrm{CO}_{2}$ produced by molasses fermentation and (i) clean sock; (ii) sock with cow odour; (iii) sock with chicken odour; (iv) sock with human odour; and, (v) MB5 blend.

The houses were mud-walled, had open eaves, and corrugated iron sheet roofs and had owner occupants throughout the night sleeping under untreated bed nets. The houses were located at least $25 \mathrm{~m}$ apart [29] to exclude the potential interaction of treatments placed in any two adjacent houses. All the baited MM-X traps were hung outside the bedroom window, under the eaves at $15 \mathrm{~cm}$ high [17].

\section{Anopheles species identification}

Adult mosquitoes were identified morphologically [30] and abdominal status was recorded (Empty (E), blood fed (F), gravid (G)) [31]. Female An. gambiae s.l. and An. funestus s.l. were preserved in 2-ml Eppendorf tubes containing $80 \%$ ethanol and a subset (215 fully blood-fed 
An. gambiae s.l. and 92 unfed An. funestus s.l.) was selected for DNA extraction (Qiagen DNeasy kit) and molecular analysis. Anopheles gambiae species were identified using a multiplex PCR approach as previously described [32], while An. funestus species were determined by PCR amplification, sequencing and phylogenetic sequence analysis of a 380-704 bp fragment of the rDNA gene using primers designed to amplify coding regions flanking the internal transcribed Spacer Region 2 (ITS2) domain [33].

\section{Blood meal identification and detection of Plasmodium}

Blood meals were identified using two PCR-based approaches. The first method utilized species-specific primers targeting a fragment (132-680 bp amplicon) of mammalian cytochrome $b$ (cytb) [34]. To ensure sensitive detection of mixed blood meals using this method, DNA from each blood fed mosquito was amplified in individual reactions, containing either a human-, cow-, goat-, pig-, or dogspecific forward primer and a universal reverse primer [34]. PCR amplicons were sequenced and subjected to phylogenetic analysis to verify blood meal origin. Second, to ensure sensitive detection of human blood meals, DNA from each mosquito was amplified using primers designed to target the hypervariable D-loop region of ape mitochondrial DNA [35], sequenced, and subjected to phylogenetic analysis.

To screen for the presence of Plasmodium parasites in field caught mosquitoes, DNA extracted from whole mosquitoes was subjected to nested PCR targeting a $956 \mathrm{bp} c y t b$ fragment of the Plasmodium mitochondrial genome [36,37]. All PCR reactions used previously reported cycling conditions and the Roche Expand Long Template PCR system.

\section{Ethical considerations}

Scientific and ethical approval of the present study was granted by the Kenya Medical Research Institute (KEMRI/ RES/7/3/1). Consent for houses used in the study was obtained from the household heads and the local administration prior to the start of the study.

\section{Statistical analysis}

A generalized linear model (GLM assuming a Binomial distribution with logit link function) was used to investigate the relative attractiveness of each combination of odours tested in the traps in the semi-field and field experiments, expressed as the number of mosquitoes of one species caught in one of the traps divided by the total number of mosquitoes of that species trapped in all traps during each experimental night $[38,39]$. The effects of treatment, position of trap or house on mosquito catches were fitted in the model and the non-significants dropped. Models were compared by the Pearson Chi-square value divided by the degrees of freedom. Differences between treatments were tested by pair-wise comparisons with least square differences (LSD) correction [40]. Effects were considered significant at $\mathrm{P}<0.05$. All analyses were performed using IBM SPSS statistical software, version 22.

\section{Results}

Attractiveness of natural host odours to laboratory-reared Anopheles gambiae and Anopheles arabiensis

Results from semi-field studies using laboratory-reared mosquitoes showed significant results among MM-X traps baited with different combinations of odours and $\mathrm{CO}_{2}$, as follows $(\mathrm{P}<0.001$, GLM, Figure 1): i) significantly higher number of both An. gambiae and An. arabiensis in traps baited with $\mathrm{CO}_{2}$ than in traps without $\mathrm{CO}_{2}$ (Additional file 1: Table $\mathrm{S} 1$ ); ii) significantly lower numbers An. gambiae and higher numbers of $A n$. arabiensis in traps baited with cow odours compared to traps with $\mathrm{CO}_{2}$ alone (Additional file 1: Table S2), iii) significantly higher numbers of An. arabiensis in traps baited with chicken odours compared to traps with $\mathrm{CO}_{2}$ alone; iv) significantly higher number of both species in traps baited with human odours compared to traps with $\mathrm{CO}_{2}$ alone.

\section{Attractiveness of natural host odours to Anopheles gambiae and Anopheles arabiensis by competition} Of 3,200 mosquitoes of each species released, 1,161 (36\%) An. gambiae and $940 \mathrm{An}$. arabiensis (29\%) were caught during the 16 experimental nights (GLM, Figure 2). The response of An. gambiae to traps baited with human odour was significantly higher than to the other treatments ( $\mathrm{P}<0.05$; GLM, Figure 2). The response of An. arabiensis was significantly higher to human odour than to cow odour or $\mathrm{CO}_{2}$ alone $(\mathrm{P}<0.001)$, and close to significant when compared to chicken odour $(\mathrm{P}=00.061$, GLM, Figure 2, Additional file 1: Table S3).

\section{Attractiveness of synthetic odour blends to Anopheles gambiae and Anopheles arabiensis}

The attractiveness of all treatments was significantly different for both mosquito species, ( $<<0.001$, GLM, Figure 3, Additional file 1: Table S4). The trap without $\mathrm{CO}_{2}$ was least attractive to mosquitoes, followed by the traps baited with $\mathrm{CO}_{2}$ alone, and then $\mathrm{CO}_{2}+\mathrm{SB}$. Traps baited with $\mathrm{CO}_{2}$ plus the MB5 blend were the most attractive to mosquitoes (GLM, Figure 3).

\section{Response of wild mosquitoes with different host preferences to natural and synthetic odour blends} A total of 6,057 wild mosquitoes were caught outdoors in Kigoche village over a period of 25 nights between May and June 2013. Of the 6,057 mosquitoes, 6\% $(n=367)$ were males and $94 \%(n=5,690)$ were females (Additional file 1: Table S5). Out of the 5,690 female mosquitoes trapped, $9 \%(\mathrm{n}=535)$ were blood fed $(\mathrm{F})$ and none was gravid (HG, G). 


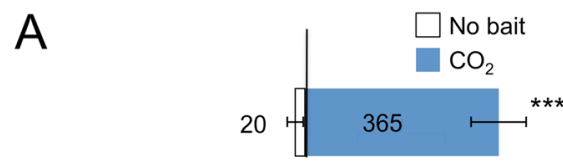

An. arabiensis

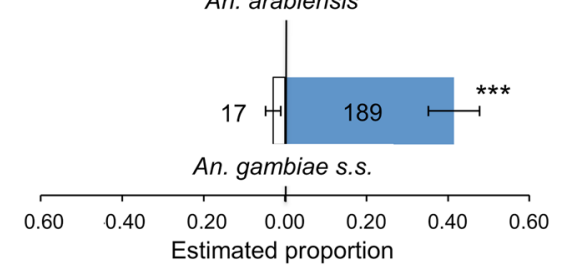

C

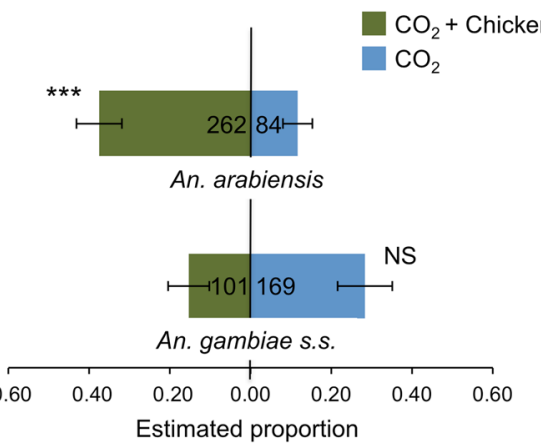

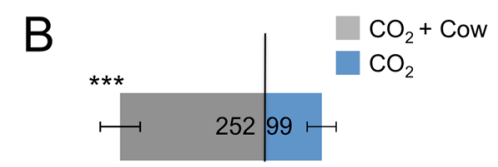

An. arabiensis
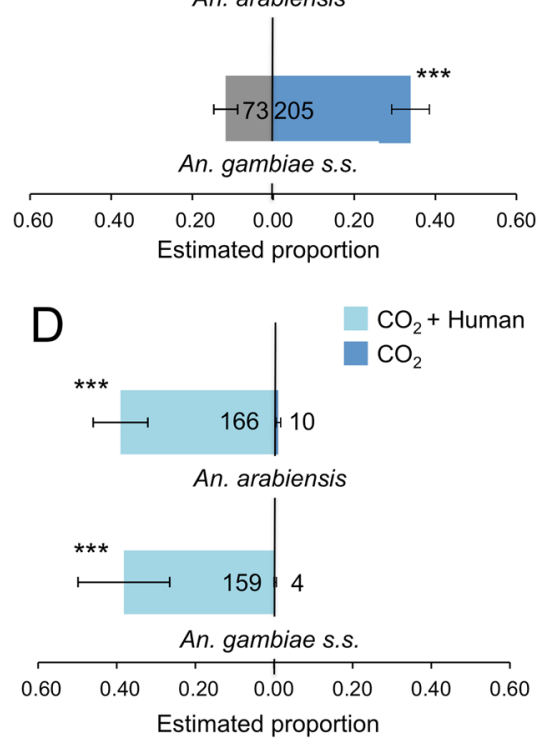

Figure 1 Screenhouse mosquito catches in dual-choice test with different odour baits. Estimated mean proportion (GLM) of mosquitoes caught in a screenhouse using MM-X traps with $\mathrm{CO}_{2}$ tested versus an empty trap (A). Cow (B) chicken $\mathbf{C}$ ) and human (D) emanations were tested in combination with $\mathrm{CO}_{2}$ versus a trap with $\mathrm{CO}_{2}$ alone. Error bars represent the standard error of the mean; ${ }^{* * *}$ : $X^{2}$-test $P<0.001$, NS: $X^{2}$-test $P>0.05$. Numbers in the bars indicate number of mosquitoes caught.

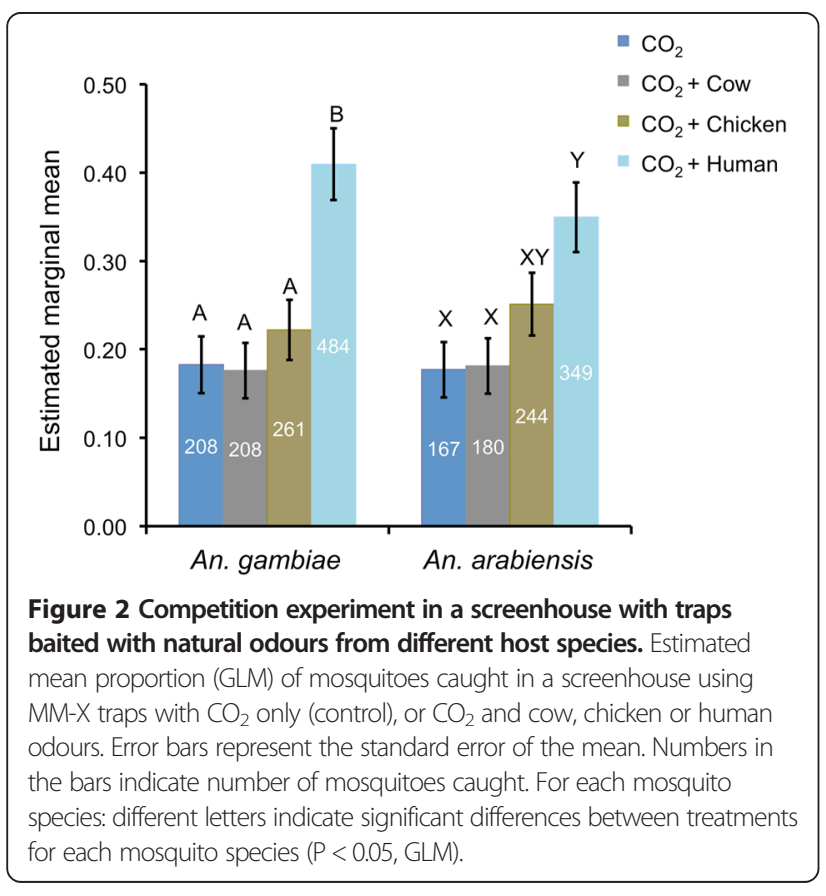

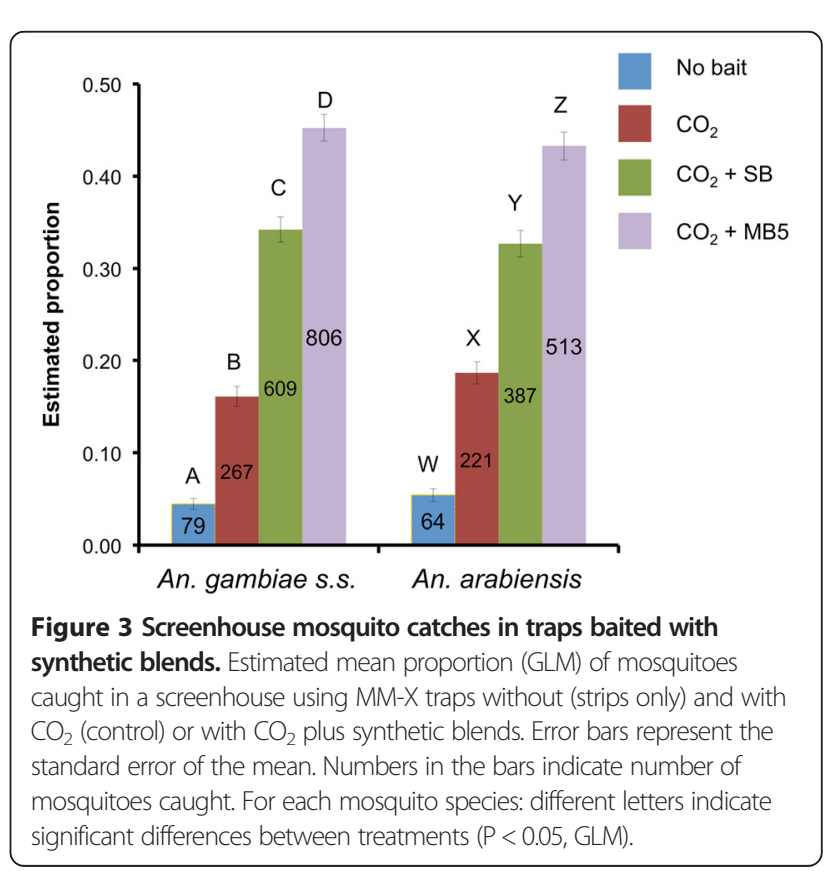


For the 'unfed' mosquitoes, $16 \%(\mathrm{n}=816)$ were $A n$. arabiensis, 23\% ( $\mathrm{n}=1,186)$ An. funestus, 35\% ( $\mathrm{n}=1,803)$ Culex spp., 20\% Mansonia spp. $(1,028)$ and $6 \%(\mathrm{n}=322)$ were other mosquito species. There was no significant difference in numbers of $A n$. arabiensis caught in traps baited with $\mathrm{CO}_{2}$ alone and traps baited with cow or chicken odours $(\mathrm{P}=00.273, \mathrm{P}=00.703$, respectively, $\mathrm{GLM}$, Figure 4A, Additional file 1: Tables S6 and S7). Human and MB5baited traps attracted equal numbers of An. arabiensis $(\mathrm{P}=00.887)$ and the catches were significantly higher than those of $\mathrm{CO}_{2}$, cow or chicken-baited traps (GLM, Figure 4A, Additional file 1: Tables S6,S7). For unfed An. funestus, $\mathrm{CO}_{2}$ and chicken odour were least attractive $(\mathrm{P}=00.696$, GLM Figure 4B, Additional file 1: Tables S6, S7). Cow or human odours were more attractive to $A n$. funestus $(\mathrm{P}=00.292)$ with higher catches than $\mathrm{CO}_{2}(\mathrm{P}=00.007)$ or chicken ( $\mathrm{P}=00.020)$ but lower than the MB5 blend which was most attractive to An. funestus $(\mathrm{P}<0.001$, GLM Figure 4B, Additional file 1: Tables S6 and S7). Compared to the response to $\mathrm{CO}_{2}$ alone, the Culex spp. did not show any enhanced attraction to the traps when natural odours or the synthetic blend were added ( $\mathrm{P}>0.05$, GLM, Additional file 1: Tables S6, S7). Mansoni spp. were more attracted to cow odour and the MB5 blend compared to traps baited with $\mathrm{CO}_{2}$ alone $(\mathrm{P}=00.010$ and $\mathrm{P}=00.007$ respectively, GLM, Additional file 1: Tables S6 and S7).

Carbon dioxide production by fermenting molasses resulted in a high percentage of blood fed mosquitoes [18]. A total of 535 out of 6,057 collected females were blood fed: 45\% were $A n$. arabiensis, $5 \%$ were $A n$. funestus, $38 \%$ were Culex spp., 8\% Mansonia spp. and 3\% were other mosquito species (Figure 4). There was a significantly higher number of blood fed An. arabiensis caught by MB5 compared to $\mathrm{CO}_{2}$, chicken or man $(\mathrm{P}<0.036)$ but not compared to cow odour baited traps $(\mathrm{P}=00.142$, GLM, Figure 4A, Additional file 1: Tables S7 and S8). Blood-fed An. funestus were caught more often in traps baited with cow odour, human odour and the MB5 blend than traps baited with $\mathrm{CO}_{2}$ alone although numbers were relatively low for a GLM analysis $(\mathrm{P}<0.021$, GLM, Figure 4B, Additional file 1: Tables S7 and S8). No significant differences were found between Culex spp. and Mansonia spp. trapped with the different treatments ( $\mathrm{P}>0.05$, GLM, Additional file 1: Tables S7 and S8).

\section{Molecular characterization of mosquitoes caught in field settings}

To confirm the species origin of wild-caught mosquitoes, 215/240 (from 25 samples the ethanol evaporated) fully blood-fed An. gambiae s.l. and a subset of 92 unfed $A n$. funestus s.l were subjected to mitochondrial DNA analysis. In concordance with previous studies [18,41], all An. gambiae s.l. were identified as An. arabiensis except for one that could not be typed due to insufficient material. Similarly, all An. funestus s.l. analysed were identified as $A n$. funestus s.s. Analysis of the same mosquitoes for the presence of Plasmodium (cytb) sequences revealed that two An. funestus were positive for Plasmodium falciparum and one An. funestus was positive for Plasmodium malariae (Additional file 1: Figure S3). None of the An. arabiensis was Plasmodium sequence positive. Blood meal analysis revealed that the vast majority of blood fed $A n$. arabiensis contained cow blood (86\%), with a small minority also containing human blood (2\%) as determined by sequence analysis of mitochondrial PCR amplicons (Table 1, Additional file 1: Figure S2). Additionally, one human, one caprid and one canine blood meal were identified. Twenty-nine $A n$. arabiensis did not yield blood meal PCR amplicons by either method.

\section{Discussion}

$\mathrm{CO}_{2}$ has been identified as an attractant for many mosquito species $[5,6,27,42,43]$. The semi-field experiments
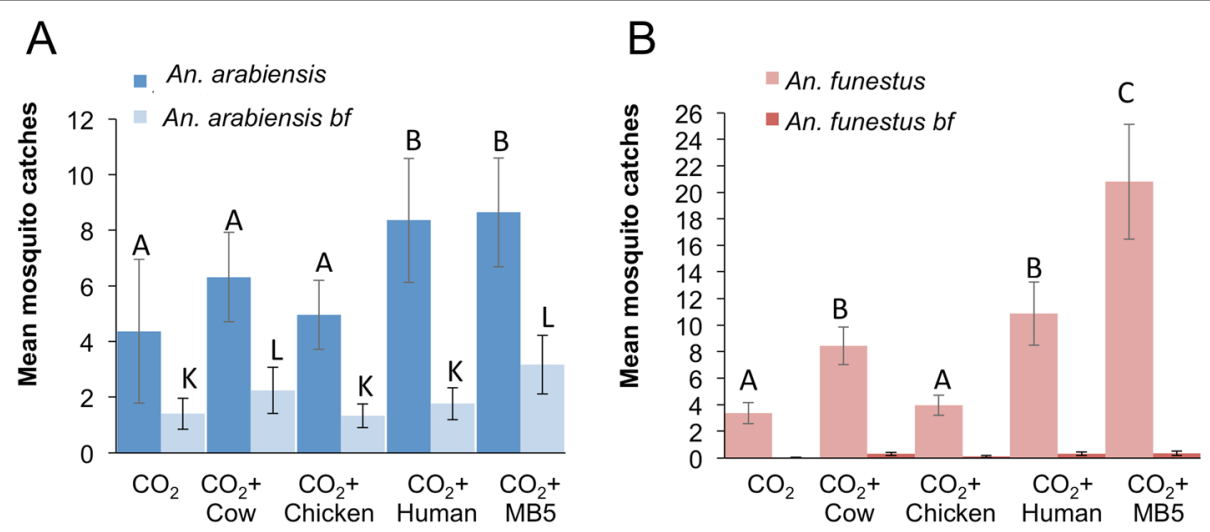

Figure 4 Mosquito catches in traps baited with natural and synthetic odours in a field set-up. Estimated mean proportion (GLM) of wild unfed or blood-fed mosquitoes caught outdoors. A) An. arabiensis, B) An. funestus s.l. caught outdoors using $\mathrm{MM}-\mathrm{X}$ traps with $\mathrm{CO}_{2}$ or $\mathrm{CO}_{2}$ and treatments. Numbers in the bars indicate number of mosquitoes caught during 25 experimental nights. For each mosquito species: different letters indicate significant differences between treatments $(P<0.05, G L M)$. 
Table 1 Blood meal identification in field caught $\mathbf{A n}$. arabiensis

\begin{tabular}{ll}
\hline Blood meal origin & No. (percent) \\
\hline Single species blood meal & $180(83.3)$ \\
Cow & $1(0.5)$ \\
Caprid & $1(0.5)$ \\
Dog & $1(0.5)$ \\
Human & \\
Multiple species blood meal & $4(1.8)$ \\
Cow and human & $29(13.4)$ \\
Blood meal undetectable &
\end{tabular}

presented here confirm that $\mathrm{CO}_{2}$ is an important cue for both An. gambiae s.s. and An. arabiensis [5,6] and that including $\mathrm{CO}_{2}$ in monitoring traps increases their efficacy (Figure 1). Adding host odours to $\mathrm{CO}_{2}$ increased trap catches for $A n$. arabiensis, but results were less clear-cut for An. gambiae. Human odour was highly attractive to both species (Figures 1 and 2) and although this has been reported previously for An. gambiae in both field and laboratory studies [1,25,44], only a few studies have reported An. arabiensis to be more attracted to human compared to cow odour [1,45-47]. The results show that An. arabiensis is opportunistic in nature. Moreover, human odour appeared to be more important than cow or chicken odour in the attractiveness to female An. arabiensis, although individual differences in attractiveness could have played a role.

Interestingly, in some of the semi-field experiments, adding cow or chicken odour to traps baited with $\mathrm{CO}_{2}$ decreased the number of An. gambiae s.s. caught (Figure 1). This effect has been reported before when $\mathrm{CO}_{2}$ was added to cow odour in an olfactometer, however, when only cow odour without $\mathrm{CO}_{2}$ was present, the inhibiting effect was not observed [25]. A field study by Costantini et al. [48] also indicated an aversion of An. gambiae s.s. to cattle odour when using odour-baited entry traps. These studies and the results presented here further confirm the anthropophilic nature of this mosquito species and the importance of both human odour and $\mathrm{CO}_{2}$ in its host-seeking behaviour.

The MB5 blend has proven to be an effective synthetic blend for monitoring malaria mosquitoes [13,14]. However, it was not clear from previous studies whether this blend would attract different species equally, and whether the host preference of these species would affect their preference to these blends. Results of semi-field experiments show that An. gambiae s.s. and An. arabiensis host preferences do not influence their response to the MB5 blend, which is attractive for both species. Nevertheless, a substantial proportion of the mosquitoes did not get trapped in the traps baited with either natural or synthetic odours and it is unclear if these mosquitoes escaped the screenhouse, were not host seeking, were influenced by the weather or were not trapped for other reasons. Field experiments also revealed a clear difference in response between the two important malaria vectors An. funestus and $A n$. arabiensis. Although human odour and the MB5 blend attracted equal numbers of An. arabiensis, the synthetic blend attracted significantly more An. funestus s.s. than traps baited with human odour. Particular odour baits selected for monitoring purposes will therefore affect both the number of mosquitoes and the ratio between the species collected. The advantage of using the MB5 blend for monitoring is that it is standardized, highly effective (Figure 4) and long lasting (Mweresa, pers. comm.).

No Plasmodium was detected in the An. arabiensis mosquitoes analysed; however, $3.3 \%$ of the An. funestus s.s. tested were Plasmodium positive. This result may be explained by the zoophilic nature of $A n$. arabiensis and more anthropophilic behaviour of An. funestus s.s.

Molecular analyses of blood-fed An. arabiensis females indicated that $87 \%$ of the blood meals were of cow origin and only $2 \%$ of human origin. Since traps were hung outside, this result may reflect host availability rather than host preference [49]. True host preference is better evaluated using choice tests [1] as performed in the semi-field experiments; however, host choice will largely depend on the host availability in the field [1]. A previous study by Mweresa et al. [18] showed that a trap with fermenting molasses, rather than fermenting sugar, significantly increased the number of blood-fed mosquitoes caught compared to the number of unfed mosquitoes. The blood meal results presented here show that the use of fermenting molasses in a trap can catch mosquitoes that have fed outdoors, since most of the blood meals were from cows and typically cattle are kept outside human habitations. This result indicates that molasses-fermenting traps are very suitable for monitoring outdoor mosquitoes and thereby outdoor transmission.

In the last decade, indoor residual spraying (IRS) and the use of long lasting insecticidal nets (LLINs) have reduced indoor mosquito populations and thereby malaria transmission [50-53]. In areas where indoor transmission has been reduced substantially through the use of LLINs and IRS, the control of outdoor malaria has become more important and there is a need, therefore, for effective tools to monitor and reduce outdoor transmission. Outdoor odour-baited traps have become increasingly efficient for catching host-seeking mosquitoes. Nonetheless, they catch few or no blood-fed mosquitoes $[10,12]$ and methods that permit the reliable and consistent trapping of blood-fed mosquitoes outdoors are not available. The combination of fermenting molasses with selected odour baits represents an important new tool for understanding outdoor mosquito behaviour, which will be of utility to measure, 
and possibly even reduce, outdoor transmission. To eliminate malaria, targeting outdoor vectors will be essential and odour-baited traps that target both host seeking and blood fed mosquitoes could become an important tool.

Odour baits, including synthetic blends, are biased in their capture efficacy, and in addition, the traps themselves may also bias mosquito catches and the odours to which mosquitoes respond [45]. These are important consideration when monitoring or mass trapping mosquitoes, however, the use of a synthetic odour blend as an attractant in traps remains a very effective and standardized method for mosquito monitoring and possibly reduction.

\section{Additional file}

Additional file 1: Collection of host odours from legs of (A) a male human, (B) cow, and (C) from chicken. Figure S2. Blood meal analysis of wild caught An. arabiensis. Figure S3. Identification of Plasmodium falciparum and Plasmodium malariae in wild caught mosquitoes.

Table S1. Mean ( \pm SE) of mosquitoes caught in a screenhouse using MM-X traps with A) without $\mathrm{CO}_{2}$, B) cow, C) chicken and D) human odours. Table S2. Mean ( \pm SE) of mosquitoes caught in a screenhouse using MM-X traps baited with natural host odours. Table S3. P-values of pair-wise comparisons (GLM) after LSD correction, based on proportions of number of mosquitoes caught in a screenhouse by use of natural host odours. The mean difference is significant at the 0.05 level. Table S4. Mean $( \pm$ SE) of mosquitoes caught in a screenhouse using MM-X traps baited with synthetic blends. Table S5. Mean $( \pm S E)$ of wild male mosquitoes caught outdoors using MM-X traps baited with natural host or synthetic odour blends. Table S6. Mean $( \pm \mathrm{SE})$ and standard deviation (SD) of wild non-fed female mosquitoes caught outdoors using MM-X traps baited with natural or synthetic odour blends. Table S7. Pair-wise comparisons of $P$ values (GLM) based on proportions of wild mosquitoes caught in MM-X traps baited with natural and synthetic odour blends. The mean difference is significant at the 0.05 level. Table S8. Mean $( \pm \mathrm{SE})$ of wild blood-fed mosquitoes caught outdoors using MM-X traps baited with natural and synthetic odour blends.

\section{Competing interests}

The authors declare that they have no competing interests.

\section{Authors' contributions}

NOV, WT and WRM designed the study; AOB conducted the research; NOV and $\mathrm{AOB}$ analysed the data; $\mathrm{AOB}, \mathrm{WRM}, \mathrm{DEL}, \mathrm{BHH}, \mathrm{WT}$, and NOV wrote the paper, DEL and BHH performed molecular species, blood meal and Plasmodium analyses. All authors read and approved the final manuscript.

\section{Acknowledgements}

We are grateful to Mr David O Alila for providing the laboratory-reared mosquitoes used during the semi-field experiments. Mrs Margaret Ayugi and Mr Erick Ambugo are thanked for making natural host odours available. We are thankful to Dr Susan Imbahale and Mr Fred Kisanya for the laboratory facility at the Ahero Multi-purpose Development Training Institute (AMDTI) and $\mathrm{Mr}$ Charles Oketch for assistance in conducting field studies alongside Mr Philemon Omusula. Gratitude goes to household owners at Kigoche village for allowing us to conduct this study near their houses. This work was supported by a grant from the Earth and Life Sciences Foundation (Veni-ALW) of The Netherlands Organisation for Scientific Research (NWO, 863.13.012) and grants from the National Institutes of Health (R01 Al091595, T32 Al007532, P30 Al045008).

\section{Author details}

${ }^{1}$ International Centre of Insect Physiology and Ecology, PO Box 30772-00100 GPO, Nairobi, Kenya. ${ }^{2}$ Laboratory of Entomology, Wageningen University, PO Box 8031, $6700 \mathrm{EH}$, Wageningen, The Netherlands. ${ }^{3}$ Departments of Medicine and Microbiology, Perelman School of Medicine, University of Pennsylvania,
3610 Hamilton Walk, Philadelphia, PA 19104-6076, USA. ${ }^{4}$ School of Biological Sciences, University of Nairobi, PO Box 30197-00100 GPO, Nairobi, Kenya.

Received: 17 December 2014 Accepted: 1 March 2015

Published online: 28 March 2015

\section{References}

1. Takken W, Verhulst NO. Host preferences of blood-feeding mosquitoes. Annu Rev Entomol. 2013;58:433-53.

2. Costantini C, Sagnon N, Della Torre A, Coluzzi M. Mosquito behavioural aspects of vector-human interactions in the Anopheles gambiae complex. Parassitologia. 1999;41:209-17.

3. WHO. World malaria report 2013. Geneva: World Health Organization; 2013. p. 286.

4. Tirados I, Costantini C, Gibson G, Torr SJ. Blood-feeding behaviour of the malarial mosquito Anopheles arabiensis: implications for vector control. Med Vet Entomol. 2006;20:425-37.

5. Mboera LEG, Knols BGJ, Braks MAH, Takken W. Comparison of carbon dioxide-baited trapping systems for sampling outdoor mosquito populations in Tanzania. Med Vet Entomol. 2000;14:257-63.

6. Gillies MT. The role of carbon dioxide in host-finding by mosquitoes (Diptera:Culicidae): a review. Bull Entomol Res. 1980;70:525-32.

7. Murphy MW, Dunton RF, Perich MJ, Rowley WA. Attraction of anopheles (Diptera: Culicidae) to volatile chemicals in western Kenya. J Med Entomol. 2001;38:242-4

8. Njiru B, Mukabana WR, Takken W, Knols BGJ. Trapping of the malaria vector Anopheles gambiae with odour-baited MM-X traps in semi-field conditions in western Kenya. Malar J. 2006:5:39.

9. Jawara M, Awolola TS, Pinder M, Jeffries D, Smallegange RC, Takken W, et al. Field testing of different chemical combinations as odour baits for trapping wild mosquitoes in The Gambia. PLoS ONE. 2011;6:e19676.

10. Mukabana WR, Mweresa CK, Otieno B, Omusula P, Smallegange RC, Van Loon JJA, et al. A novel synthetic odorant blend for trapping of malaria and other African mosquito species. J Chem Ecol. 2012;38:235-44.

11. Smallegange RC, Qiu YT, Van Loon JJA, Takken W. Synergism between ammonia, lactic acid and carboxylic acids as kairomones in the host-seeking behaviour of the malaria mosquito Anopheles gambiae sensu stricto (Diptera: Culicidae). Chem Senses. 2005;30:145-52.

12. Okumu FO, Killeen GF, Ogoma S, Biswaro L, Smallegange RC, Mbeyela ET, et al. Development and field evaluation of a synthetic mosquito lure that is more attractive than humans. PLoS ONE. 2010;5:e8951.

13. Menger D, Van Loon J, Takken W. Assessing the efficacy of candidate mosquito repellents against the background of an attractive source that mimics a human host. Med Vet Entomol. 2014:28:407-13.

14. Hiscox A, Otieno B, Kibet A, Mweresa CK, Omusula P, Geier M, et al. Development and optimization of the Suna trap as a tool for mosquito monitoring and control. Malar J. 2014;13:257.

15. Smallegange RC, Qiu YT, Bukovinszkiné-Kiss G, Van Loon JJA, Takken W. The effect of aliphatic carboxylic acids on olfaction-based host-seeking of the malaria mosquito Anopheles gambiae sensu stricto. J Chem Ecol. 2009;35:933-43.

16. Verhulst NO, Mbadi P, Kiss G, Mukabana W, Van Loon JJA, Takken W, et al. Improvement of a synthetic lure for Anopheles gambiae using compounds produced by human skin microbiota. Malar J. 2011;10:28.

17. Verhulst NO, Mukabana WR, Takken W, Smallegange RC. Human skin microbiota and their volatiles as odour baits for the malaria mosquito Anopheles gambiae s.s. Entomol Exp Appl. 2011;139:170-9.

18. Mweresa CK, Omusula P, Otieno B, Van Loon JJ, Takken W, Mukabana WR. Molasses as a source of carbon dioxide for attracting the malaria mosquitoes Anopheles gambiae and Anopheles funestus. Malar J. 2014;13:160.

19. Verhulst NO, Loonen JACM, Takken W. Advances in methods for colour marking of mosquitoes. Parasit Vectors. 2013;6:200.

20. Bukhari T, Takken W, Koenraadt C. Development of Metarhizium anisopliae and Beauveria bassiana formulations for control of malaria mosquito larvae. Parasit Vectors. 2011;4:23.

21. Atieli H, Menya D, Githeko A, Scott T. House design modifications reduce indoor resting malaria vector densities in rice irrigation scheme area in western Kenya. Malar J. 2009;8:108.

22. Nyasembe VO, Tchouassi DP, Kirwa HK, Foster WA, Teal PEA, Borgemeister C, et al. Development and assessment of plant-based synthetic odor baits for surveillance and control of malaria vectors. PLoS ONE. 2014;9:e89818. 
23. Githeko A, Mbogo C, Atieli F, Juma F. Plasmodium falciparum sporozoite and entomological inoculation rates at the Ahero rice irrigation scheme and the Miwani sugar-belt in western Kenya. Ann Trop Med Parasit. 1993;87:379-91.

24. Mukabana WR, Takken W, Coe R, Knols BGJ. Host-specific cues cause differential attractiveness of Kenyan men to the African malaria vector Anopheles gambiae. Malar J. 2002;1:17.

25. Pates HV, Takken W, Stuke K, Curtis CF. Differential behaviour of Anopheles gambiae sensu stricto (Diptera: Culicidae) to human and cow odours in the laboratory. Bull Entomol Res. 2001;91:289-96.

26. Olanga E, Okal M, Mbadi P, Kokwaro E, Mukabana W. Attraction of Anopheles gambiae to odour baits augmented with heat and moisture. Malar J. 2010;9:6

27. Smallegange R, Schmied W, Van Roey K, Verhulst NO, Spitzen J, Mukabana $W$, et al. Sugar-fermenting yeast as an organic source of carbon dioxide to attract the malaria mosquito Anopheles gambiae. Malar J. 2010;9:292.

28. Schmied WH, Takken W, Killeen GF, Knols BGJ, Smallegange RC. Evaluation of two counterflow traps for testing behaviour-mediating compounds for the malaria vector Anopheles gambiae s.s. under semi-field conditions in Tanzania. Malar J. 2008;7:230.

29. Hill N, Lenglet A, Arnez AM, Carneiro I. Plant-based insect repellent used in combination with insecticide treated bed nets give greater protection against malaria than treated nets alone in areas of early evening biting vectors: a double-blind, placebo controlled clinical trial in the Bolivian Amazon. BMJ. 2007;335:1023.

30. Gillies MT, Coetzee M. A supplement to the Anophelinae of Africa South of the Sahara. Johannesburg: The South African Institute for Medical Research; 1987.

31. WHO. Malaria entomology and vector control. Geneva: World Health Organization; 2013.

32. Scott JA, Brogdon WG, Collins FH. Identification of single specimens of the Anopheles gambiae complex by the polymerase chain reaction. Am J Trop Med Hyg. 1993:4:520-9.

33. Koekemoer $L$, Kamau L, Hunt R, Coetzee M. A cocktail polymerase chain reaction assay to identify members of the Anopheles funestus (Diptera: Culicidae) group. Am J Trop Med Hyg. 2002;66:804-11.

34. Kent RJ, Norris DE. Identification of mammalian blood meals in mosquitoes by a multiplexed polymerase chain reaction targeting cytochrome $\mathrm{B}$. Am J Trop Med Hyg. 2005;73:336-42.

35. Gao F, Bailes E, Robertson DL, Chen Y, Rodenburg CM, Michael SF, et al. Origin of HIV-1 in the chimpanzee Pan troglodytes troglodytes. Nature. 1999:397:436-41.

36. Prugnolle F, Durand P, Neel C, Ollomo B, Ayala FJ, Arnathau C, et al. African great apes are natural hosts of multiple related malaria species, including Plasmodium falciparum. Proc Natl Acad Sci U S A. 2010;107:1458-63.

37. Liu W, Li Y, Learn GH, Rudicell RS, Robertson JD, Keele BF, et al. Origin of the human malaria parasite Plasmodium falciparum in gorillas. Nature. 2010;467:420-5.

38. Qiu YT, Smallegange RC, Van Loon JJA, Ter Braak CJF, Takken W. Interindividual variation in the attractiveness of human odours to the malaria mosquito Anopheles gambiae s.s. Med Vet Entomol. 2006;20:280-7.

39. Verhulst NO, Qiu YT, Beijleveld H, Maliepaard C, Knights D, Schulz S, et al. Composition of human skin microbiota affects attractiveness to malaria mosquitoes. PLoS One. 2011;6:e28991.

40. Pombi M, Jacobs F, Verhulst NO, Caputo B, Della Torre A, Takken W. Field evaluation of a novel synthetic odour blend and of the synergistic role of carbon dioxide for sampling host-seeking Aedes albopictus adults in Rome, Italy. Parasit Vectors. 2014;7:580.

41. Menger DJ, Otieno B, De Rijk M, Mukabana WR, Van Loon JJ, Takken W. A push-pull system to reduce house entry of malaria mosquitoes. Malar J. 2014;13:119.

42. Reeves WC. Field studies of carbon dioxide as a possible host simulant to mosquitoes. Proc Soc Exp Biol Med. 1951;77:64-6.

43. Russell RC. The relative attractiveness of carbon dioxide and octenol in CDC-and EVS-type light traps for sampling the mosquitoes Aedes aegypti (L.), Aedes polynesiensis Marks, and Culex quinquefasciatus say in Moorea, French Polynesia. J Vector Ecol. 2004;29:309-14.

44. Gillies MT. Selection for host preference in Anopheles gambiae. Nature. 1964;203:852-4.

45. Torr SJ, Della Torre A, Calzetta M, Costantini C, Vale GA. Towards a fuller understanding of mosquito behaviour: use of electrocuting grids to compare the odour-orientated responses of Anopheles arabiensis and An. quadriannulatus in the field. Med Vet Entomol. 2008;22:93-108.

46. Diatta M, Spiegel A, Lochouarn L, Fonyenille D. Similar feeding preferences of Anopheles gambiae and An. arabiensis in Senegal. Trans R Soc Trop Med Hyg. 1998;92:270-2.

47. Lyimo IN, Haydon DT, Russell TL, Mbina KF, Daraja AA, Mbehela EM, et al. The impact of host species and vector control measures on the fitness of African malaria vectors. Proc Royal Society B: Biol Sci. 2013;280:1-10.

48. Costantini C, Sagnon N, Della Torre A, Diallo M, Brady J, Gibson G, et al. Odor-mediated host preferences of West-African mosquitoes, with particular reference to malaria vectors. Am J Trop Med Hyg. 1998;58:56-63.

49. Lyimo IN, Ferguson HM. Ecological and evolutionary determinants of host species choice in mosquito vectors. Trends Parasitol. 2009;25:189-96.

50. Pluess B, Tanser FC, Lengeler C, Sharp BL. Indoor residual spraying for preventing malaria. Cochrane Database Syst Rev. 2010;4:CD006657.

51. Lengeler $\mathrm{C}$. Insecticide-treated bed nets and curtains for preventing malaria. Cochrane Database Syst Rev. 2004;2:CD000363.

52. Bayoh MN, Mathias D, Odiere M, Mutuku F, Kamau L, Gimnig J, et al. Anopheles gambiae: historical population decline associated with regional distribution of insecticide-treated bed nets in western Nyanza Province, Kenya. Malar J. 2010;9:62.

53. Russell TL, Govella NJ, Azizi S, Drakeley CJ, Kachur SP, Killeen GF. Increased proportions of outdoor feeding among residual malaria vector populations following increased use of insecticide-treated nets in rural Tanzania. Malar J. 2011;10:80.

\section{Submit your next manuscript to BioMed Central and take full advantage of:}

- Convenient online submission

- Thorough peer review

- No space constraints or color figure charges

- Immediate publication on acceptance

- Inclusion in PubMed, CAS, Scopus and Google Scholar

- Research which is freely available for redistribution 